\title{
A Case Study on Graphic Path Analysis Using Various Mapping Techniques
}

\author{
${ }^{1}$ Prasad Layam, ${ }^{2}$ Dr. Vitushi Sarma \\ ${ }^{12}$,Research Scholor, Professor, ECE, University of Allahabad
}

\begin{abstract}
This work offers a method for the visual-based navigation of a mobile robot in indoor environments, using a single omni-directional camera. The geometry of the catadioptric sensor and the method used to obtain a bird's eye (orthographic) view of the ground plane are presented. This picture evocatively abridges the solution to steering problems, by eliminating any perspective effects. The nature of each navigation task is taken hooked on account when conniving the compulsory navigation skills and environmental representations. Topological Navigation is cast-off for itinerant long distances plus does not require knowledge of the exact location of the robot but rather, a qualitative position happening the topological map. For indoor robots in structured environments, we consume owed separately with the cases of geometrical and topological models of interplanetary. For shapeless environments, we consume discussed the cases of navigation through optical activities, using methods from the appearance-based paradigm, and by recognition of specific substances in the environment. The navigation process cartels appearance based approaches and visual serving upon some environmental features. Graphic Path Succeeding is obligatory for native, actual precise navigation, for e.g. door traversal, docking. The robot is controlled to follow a pre-specified trail accurately, by tracking visual milestones in bird's eye views of the ground plane. By clearly separating the nature of these triangulation tasks, a simple besides yet powerful navigation scheme is obtained.
\end{abstract}

Keywords: Navigation, robot, bird eye, orthographic, environment, triangulation

\section{Introduction}

Panoramic/omnidirectional representations of image sequences have a wide application scope, including robot navigation, virtual reality, interactive 2D/3D video, content-based video compression, and fullview video surveillance. Scene modeling using image mosaicing and panoramic/omnidirectional vision has attracted great attentions in the fields of computer vision and computer graphics in recent years. Usually, researchers either focus on the analysis/recognition part (e.g., panoramic /omnidirectional vision for robot navigation), or on the synthesis/visualization part (e.g. image mosaicing, panoramic and layered representation in image-based rendering or virtualized reality). This thesis makes a first attempt to systematically bring the two seemingly quite different topics under a single umbrella of "visual modeling and presentation".

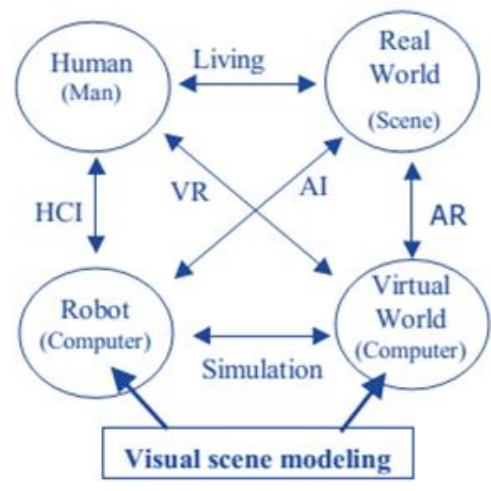

Fig 1.0 Interactions diagrams

First, let us have a look at the two topics - robot navigation and virtualized reality -- in a broader perspective of interaction between "being" and "environment" (Fig. 1). We can find a very close resemblance between them: robot navigation is the interaction between a robot (i.e. a digital being) and the real 3D world, while virtual/virtualized reality is the interaction between a person (i.e. a human being) and a virtual/virtualized environment.

Second, a closer examination of the research efforts of the past ten years shows that techniques and representations for the two applications are surprisingly similar. Graphics people talk about multiperspective projection for image-based rendering of large-scale scenes, while vision people try to use the concept of spatio- 
temporal panoramic view images in robot localization and landmark recognition. Vision/robotics people take advantage of the 360-degree view angle of omnidirectional images for map building, road following, obstacle detection in robot navigation, whereas graphics people try to generate omnidirectional image representations for image-based rendering. My own research also shows that we can use the exact same basic methodology for building and the same structures for representing visual scene models for both robot navigation and image-based rendering.

Needless to say, visual navigation of a mobile robot in a natural environment has always been a very interesting but challenging problem. It involves almost every aspect of computer vision research - from visual sensors through robust algorithms to visual representations. Another challenge in writing a survey on computer vision for mobile robot navigation is that even the perception of what constitutes progress varies widely in the research community. To us, for a mobile robot to engage in vision-based hallway navigation in the kinds of environments shown in Fig. 1 represents significant progress for the entire research community. But, others would pooh-pooh such an accomplishment on various grounds.

To the extent that successful attempts at vision-based navigation in the cluttered environments of the kind shown in the figure use underlying models of space that are highly geometrical, the "nonbelievers" could question the "nonhuman" nature of these models. The nonbelievers would add — and do so rightly - that such representations would be difficult to extend to other scenarios where a robot may need to seek out certain objects in the environment on the basis of their semantic significance in the presence of competing clutter. The basic requirements of visual navigation include global localization (to decide where to go), road following (to stay on the road) and obstacle detection (to avoid collision). Only after these safety requirements have been satisfied, which have been proven to be not a trivial problem, can the robot pursue other task-oriented goals. It is clear that visual environment modelling is the foundation of these basic issues in visual navigation - and it may extend to most of the real world problems in computer vision.

\section{Concept}

In particular, interior space was represented by CAD models of varying complexity. In some of the reported work [20], the CAD models were replaced by simpler models, such as occupancy maps, topological maps or even sequences of images. When sequences of images were used to represent space, the images taken during navigation were submitted to some kind of appearance-based matching between the perception (actual image) and expectation (goal image or goal images stored in the database). In order to apply these two kinds of compact representations to an easily-captured image sequence, we have proposed and implemented a two-stage approach. In the first stage, a 3D image stabilization method is proposed to de-couple vibrating motion and scene structure, thus making the epipolar plane image analysis and the panoramic view image representation valid for unstabilized image sequences. Notice that a commercial off-the-shelf camcorder with a digital stabilizing function usually distorts the perspective geometry of an image sequence because it uses 2D translation to stabilize the video sequence. Existing digital stabilization algorithms (e.g., Hansen, et al., 1994; Morimoto and Chellappa, 1997) are not designed to meet the need of keeping the loci straight in an EPI for a long image sequence. Our 3D stabilization algorithms with 3D image warping and motion filtering are specially designed for EPI generation and 3D recovery. Three algorithms are proposed for motion filtering, namely the locus tracking and fitting approach, motion classification and selection approach and statistical locus smoothing/fitting approach. Experimental results on many real image sequences have shown that seamless PVI and EPI can be generated.

In the second stage, a Fourier energy method over a large Gaussian-windowed area of an EPI is proposed to robustly detect multiple orientations of the EPI's motion texture in the frequency domain.

\section{The Vehicular Motion Model}

Suppose that a camera is mounted on a vehicle moving on an approximately flat road surface. In order to construct the 3D model of a roadside scene, the camera's optical axis is perpendicular to the motion direction and its horizontal axis is parallel to the motion direction 1 . Within a considered long time period $[0, \mathrm{~T}]$, we assume that the motion of the vehicle (camera) consists of a smooth planar motion and an unpredictable small fluctuation due to the vehicle's motion over a rough surface. In many real cases, the smooth motion can be approximated as a constant velocity $(\mathrm{V})$ translation. The small fluctuation between two successive frames is modeled by three small rotation angles $\Omega \mathrm{x} \Omega \mathrm{y} \Omega \mathrm{z}$, , around the X, Y and $\mathrm{Z}$ axes and three translation components along the three axes (Fig. 1). A generalization of this model Tx , Ty, Tz is given. 


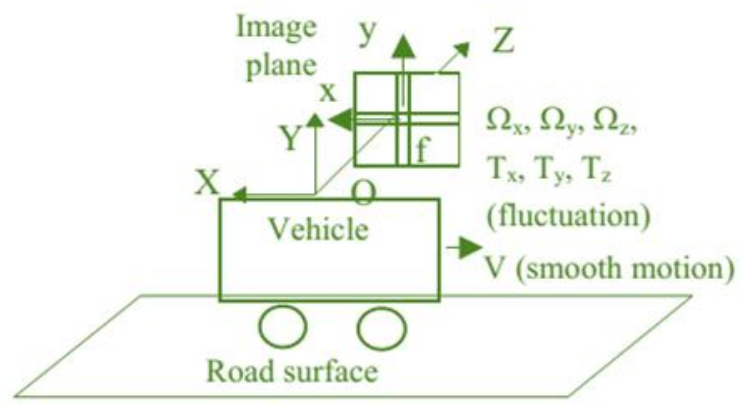

Fig 2.0 The vehicular motion model

\section{Image Rectification}

The aim of image rectification is to generate an image sequence that has horizontally parallel motion parallax between each pair of successive frames.

\section{Motion Filtering Algorithms}

Even if the motion parallax field becomes a horizontal parallel field in the $\mathrm{x}$ direction between two successive frames, the locus of a feature point across multiple frame may not be a straight line due to motion model approximation and the decomposition. Note that $\mathrm{m}(\mathrm{ti})$ can also form a parallel field by adding any constant value (instead of $\mathrm{a}^{\prime}$ ) to the motion parallax of each point $\mathrm{i}$. To remove this ambiguity, in principle, we need to assume that a specific feature point $i$ can be tracked across multiple frames $(t=0,1, \ldots, T)$ in the image sequence. Then the task of motion filtering is to find a straight line out of point set $\left\{\left(\mathrm{m}^{\wedge} \mathrm{t}(\mathrm{i}), \mathrm{t}\right)(\mathrm{t}=0,1, \ldots, \mathrm{T})\right\}$, which satisfies the assumption of a 1D translational motion with the constant speed V. The procedure of the motion filtering is to find a small shift qt for each frame so that the shifted image.

\section{Adaptive EPI Analysis Approach}

Spatio-temporal panoramic view images (PVIs) provide a compact representation for large-scale scene. Stereo PVIs can be used to estimate the depth information of the scene. The difference between panoramic stereo and the traditional stereo is that panoramic images are parallel-perspective projections. The depth of a point is proportional to the "displacement" in the t direction in a pair of stereo PVIs arefixed and D $\propto \mathrm{dt}$ ), which means that depth resolutions are the same for different depths. However, there are some disadvantages when we use stereo PVIs to recover the depth of a scene. First, stereo PVI approach faces the same correspondence problem as in any traditional stereo methods. Second, occluding regions in two panoramic views cannot be easily handled due to the lack of information. The solution to these two problems is to effectively use the information in between, i.e. that of the epipolar plane images. Our panoramic epipolar plane analysis method consists of three important parts: orientation detection, motion boundary localization and occlusion recovery. It has the following three advantages. It is roust applying to a complex natural scene and a motion with fluctuation. With a spatio-temporal-frequency domain analysis, no feature detection, hard thresholding and locus tracking are used in our algorithm. It is efficient in that it only processes a small fraction of the necessary data instead of the entire 3D ST images.

\section{Real-Time Obstacle Detection}

The non-zero disparity filter (NZD filter) is the heart of the RTSOD algorithm. In Coombs \& Brown's binocular active vision system [3], the 3D shape of the horopter (surface of zero stereo disparity) is rather complicated (and may not be well defined), since it involves vertical, as well as horizontal, disparities. In the RTSOD system, the gaze transformation creates a zero disparity plane for all the features on the ground plane. In this way obstacles can be detected easily by the NZD filter. The image difference operation on a stereo pair after gaze transformation separates points with non-zero disparities from those with zero disparities by examining difference values. Theoretically, all the pixels of ground plane features will have zero values in the difference image. However, in practice, this property can not be kept strictly due to the photo-metric and geometric bias. So the NZD Filter is applied to throw away points whose difference values are lower than a certain threshold, and then to filter out small areas which imply small disparities. A grouping procedure gathers non-disparity points into each possible obstacle regions.

The basic algorithm consists of six steps:

Step 1. Binocular imaging: An image pair of left and right images Li and Ri is captured simultaneously by the single camera binocular imaging system (I or II) and a frame grabber. 
Step 2. Gaze transformation: A reprojected left image Lpi is created by re-projecting the left image Li to the plane of the right image Ri .

Step 3. Image difference: Difference image Di is produced by calculate the absolute difference of each corresponding points in Lpi and Ri

Step 4. Non-zero disparity filtering: Non-zero disparity image Bi is created by first throwing away the points in the difference image Di whose value is smaller than a certain threshold $\mathrm{T}$, then filtering out small areas which indicate small disparities.

Step 5. Obstacle grouping: A set of obstacle regions $\{\mathrm{Oi}, \mathrm{i}=1, \mathrm{LN}\}$ is obtained by grouping connected or nearby points into each obstacle region Oi. Sizes of obstacles and distances between obstacles are considered in the grouping procedure.

Step 6. Parameter Computing. For each obstacle (region), location ( $\mathrm{x}, \mathrm{y}$ ), width $\mathrm{w}$ and height $\mathrm{h}$ are calculated and an uncertainty value is estimated.

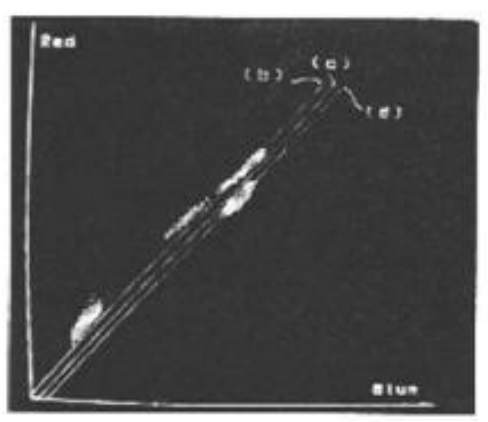

\section{Outputs}
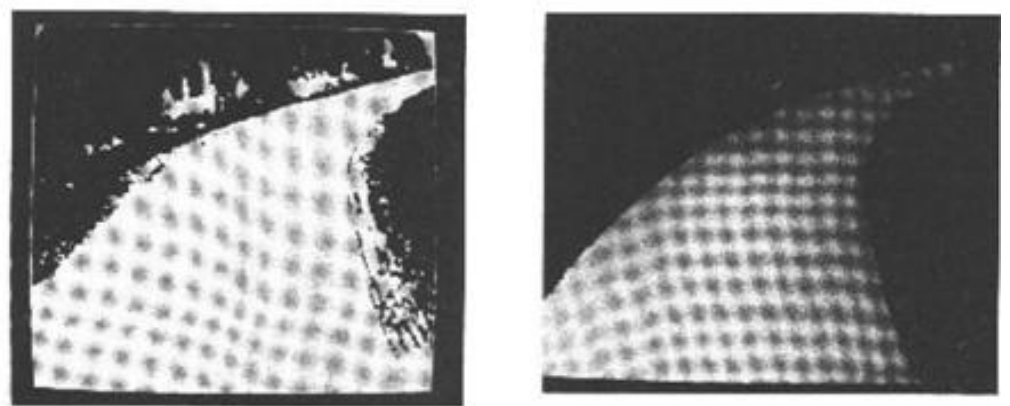

Fig 3.0 The segmentation results for different thresholding values

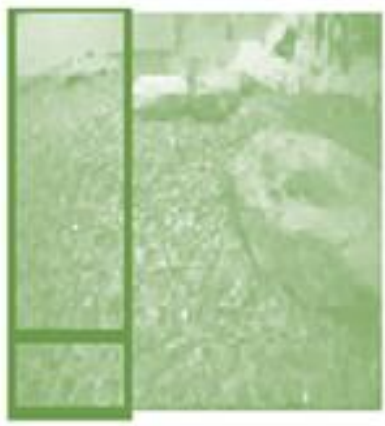

(a)

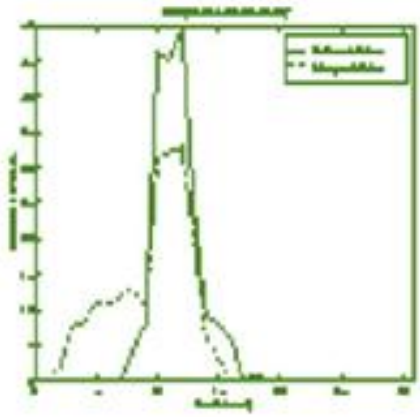

(c)

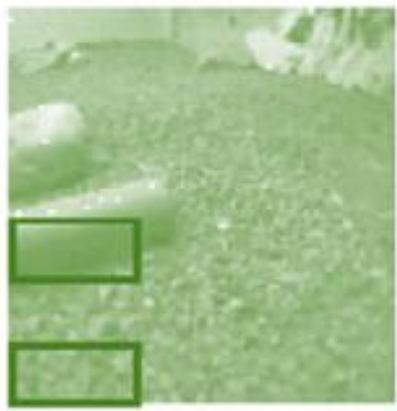

(b)

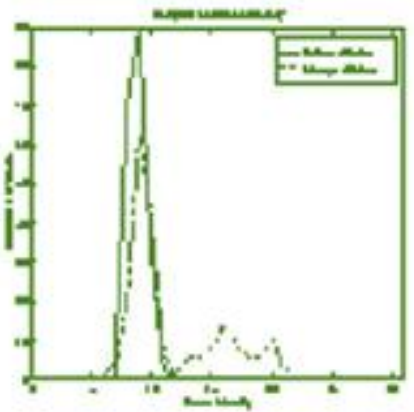

(d)

Fig 4.0 Vision-guided obstacle avoidance in [91]. (a) The camera image and the superimposed vertical slice with the safe window at the bottom.

(b) Highlights a horizontal slice and the safe window. (c) and (d) Shows the histogram of the areas highlighted in (b) 


\section{Conclusion}

In the basic RTSOD algorithm, the operation condition is that the vehicle moves smoothly, i.e. the tilted angle due to the vehicle's bump is smaller than the tolerance value. So that the gaze transformation can be realized with look-up tables with known camera posture parameters. However mistakes may occur if the vehicle bumps severely on a textured road surface. In this case the tilted angle should be measured whenever necessary in order to modify the parameters of the gaze transformation. We have proposed the dynamic gaze transformation algorithm, which can be implemented in near real time. The models can be geometrical, topological, appearance-based, etc. Different representation schemes vary with respect to the degree of metrical knowledge contained, exhibiting different degrees of robustness with regard to illumination variations, etc. This paper has surveyed these various aspects of the progress made so far in vision for mobile robot navigation. The basic idea is to establish the relation between the tilted angle and the gaze parameters, and find the best parameters for zero-disparities of the ground plane. The single-camera stereo equipment and the obstacle detection system can be used in smart cars, traffic monitoring, surveillance, virtual reality and medical imaging. The novel gaze transformation is a powerful pre-processing method for 3D scene modelling and motion analysis. It can greatly simplify the correspondence problem of stereo vision and the tracking problem in visual motion.

\section{References}

[1]. R.C. Arkin, "Motor Schema-Based Mobile Robot Navigation: An Approach to Programming by Behavior," Proc. 1987 IEEE Int'l Conf. Robotics and Automation, pp. 264-271, 1987.

[2]. R.C. Arkin, "Motor Schema-Based Mobile Robot Navigation,” Int'1 J. Robotics Research, vol. 8, no. 4, pp. 92 -112, 1989.

[3]. R. C. Arkin, "Temporal Coordination of Perceptual Algorithms for Mobile Robot Navigation," IEEE Trans. Robotics and Automation, vol. 10, no. 3, pp. 276-286, June 1994

[4]. S. Atiya and G.D. Hager, "Real-Time Vision-Based Robot Localization," IEEE Trans. Robotics and Automation, vol. 9, no. 6, pp. 785-800, Dec. 1993.

[5]. Artifical Vision for Mobile Robots: Stereo Vision and Multisensory Perception, N. Ayache and P.T. Sander, eds. MIT Press, 1991.

[6]. N. Ayache and O. D. Faugeras, "Maintaining Representations of the Environment of a Mobile Robot," IEEE Trans. Robotics and Automation, vol. 5, no. 6, pp. 804-819, 1989 .

[7]. P. Backes, K. Tso, and K. Tharp, "Mars Pathfinder Mission Internet-Based Operations Using WITS," Proc. 1998 IEEE Int'l Conf. Robotics and Automation, vol. 1, pp. 284-291, May 1998.

[8]. C. Balkenius, "Spatial Learning with Perceptually Grounded Representations," Robotics and Autonomous Systems, vol. 5, no. 34, pp. 165-175, Nov. 1998. 